\title{
Vortices in a rotating Bose-Einstein condensate: Critical angular velocities and energy diagrams in the Thomas-Fermi regime
}

\author{
Amandine Aftalion* \\ Laboratoire d'Analyse Numérique, B. C. 187, Université Paris 6, 175 Rue du Chevaleret, 75013 Paris, France \\ Qiang $\mathrm{Du}^{\dagger}$ \\ Department of Mathematics, Hong Kong University of Science and Technology, Clear Water Bay, Hong Kong
}

(Received 14 March 2001; published 8 November 2001)

\begin{abstract}
For a Bose-Einstein condensate placed in a rotating trap and strongly confined along the $z$ axis, we set a framework of study for the Gross-Pitaevskii energy in the Thomas-Fermi regime for an effective twodimensional (2D) situation in the $x-y$ plane. We investigate an asymptotic expansion of the energy, the critical angular velocities of nucleation of vortices with respect to a small parameter $\varepsilon$, and the location of vortices. The limit $\varepsilon$ going to zero corresponds to the Thomas-Fermi regime. The nondimensionalized energy is similar to the Ginzburg-Landau energy for superconductors in the high- $\kappa$ high-field limit and our estimates rely on techniques developed for this latter problem. We also take advantage of this similarity to develop a numerical algorithm for computing the Bose-Einstein vortices. Numerical results and energy diagrams are presented.
\end{abstract}

DOI: 10.1103/PhysRevA.64.063603

PACS number(s): 03.75.Fi, 02.70.-c

\section{INTRODUCTION}

Since the first experimental achievement of Bose-Einstein condensates in atomic gases, many properties of these systems have been studied experimentally and theoretically, and particularly the existence of vortices [1-10]. Two different groups have obtained vortices experimentally in atomic condensates. In Ref. [7] optical engineering of the experiment of Refs. [8] and [9] is used; a laser beam rotating with angular velocity $\widetilde{\Omega}$ is imposed on the magnetic trap holding the atoms to create a harmonic anisotropic potential. Vortex nucleation was observed [8] as well as vortex arrays [9]. Let us mention another type of experiment [11] where a laser beam is stirred in a condensate. There is a critical velocity under which the flow is dissipationless. Related analysis is given in [12]. Here, we will be concerned with the experiment of Refs. $[8,9]$.

Theoretical studies of this type of experiment have often been made in the framework of the nonlinear GrossPitaevskii equation, well known for superfluids, but which provides a very good description of Bose-Einstein condensates: it is assumed that the $N$ particles of the gas are condensed in the same state for which the wave function $\phi$ minimizes the Gross-Pitaevskii energy. By introducing a rotating frame for the angular velocity $\widetilde{\mathbf{\Omega}}=\widetilde{\Omega} \mathbf{e}_{z}$, the trapping potential becomes time independent, and the wave function $\phi$ minimizes the energy

$$
\begin{aligned}
\mathcal{E}_{3 D}(\phi)= & \int \frac{\hbar^{2}}{2 m}|\boldsymbol{\nabla} \phi|^{2}+\frac{m}{2} \sum_{\alpha} \omega_{\alpha}^{2} r_{\alpha}^{2}|\phi|^{2}+\frac{N}{2} g_{3 D}|\phi|^{4} \\
& -\hbar \widetilde{\mathbf{\Omega}} \cdot(i \phi, \boldsymbol{\nabla} \phi \times \mathbf{x}),
\end{aligned}
$$

under the constraint $\int|\phi|^{2}=1$. Here, for any complex quan-

\footnotetext{
*Electronic address: aftalion@ann.jussieu.fr

†Electronic address: madu@ust.hk
}

tities $u, v$ and their complex conjugates $\bar{u}, \bar{v},(u, v)=(u \bar{v}$ $+\bar{u} v) / 2$. The terms in the energy correspond to the kinetic energy, the trapping potential energy, the interaction energy, and the inertia due to the change of frame.

We consider a situation in which the confinement along the $z$ axis is so strong that the motion of particles along this axis is frozen in the ground state of the strong harmonic potential: hence the wave function $\phi$ is factorized into a component purely dependent on $z$ and another one $\psi$ that depends on the radial coordinates $(x, y)$ and minimizes

$$
\begin{aligned}
\mathcal{E}_{2 D}(\psi)= & \int \frac{\hbar^{2}}{2 m}|\nabla \psi|^{2}+\frac{m}{2} \sum_{\alpha=x, y} \omega_{\alpha}^{2} r_{\alpha}^{2}|\psi|^{2}+\frac{N}{2} g|\psi|^{4} \\
& -\hbar \widetilde{\mathbf{\Omega}} \cdot(i \psi, \nabla \psi \times \mathbf{\nabla}),
\end{aligned}
$$

where $g=g_{3 D}\left(m \omega_{z} / 2 \pi \hbar\right)^{1 / 2}$. The constraint $\int|\psi|^{2}=1$ is also imposed. Our study was originally motivated by the work of Castin and Dum [2], who studied the equilibrium configurations by looking for the minimizers in a reduced class of functions for the two-dimensional (2D) case and did numerical computations in 2D and 3D. Their analysis is in the Thomas-Fermi regime, where the mean interaction energy per particle is larger than $\hbar \omega_{x, y}$.

Our aim is to provide a mathematical framework for a rigorous study of the energy $\mathcal{E}_{2 D}$ and its minimizers in the Thomas-Fermi limit. We first observe that this energy has a striking similarity with the high- $\kappa$, high-field limit of the Ginzburg-Landau free energy used in the modeling of superconductors. Thus, we expect the energy $\mathcal{E}_{2 D}$ will develop similar behavior to that for the Ginzburg-Landau energy studied in [13-15]. In particular, the results obtained in the context of the Ginzburg-Landau energy may be applied to $\mathcal{E}_{2 D}$, in the Thomas-Fermi regime, to yield an asymptotic expansion of the energy as well as the critical angular velocities for the nucleation of vortices and the location of these 
vortices. Due to the close resemblance, we will not be concerned with detailed derivations in this paper, but rather focus on the conclusions one can draw from the asymptotic expansions. To our knowledge, some of our estimates to be presented later have not been given in the literature previously. Our method of computing the energy is also very different from the ones in, for instance, $[2,4,10]$.

Let $d=\left(\hbar / m \omega_{x}\right)^{1 / 2}$ be the characteristic length, and

$$
\varepsilon^{2}=\hbar^{2} /(2 N g m) .
$$

In the Thomas-Fermi approximation, $\varepsilon$ is small, which will be our asymptotic regime. In the experiment of Madison et al., $\varepsilon$ is of the order of $10^{-2}$. In fact, $\varepsilon$ characterizes the vortex size. We rescale the distance by $R=d / \sqrt{\varepsilon}$ and define $u(\mathbf{r})=R \psi(\mathbf{x})$ where $\mathbf{x}=R \mathbf{r}$. We assume that $\omega=\omega_{x}$ and $\omega_{y}$ $=\lambda \omega$ with $0 \leqslant \lambda \leqslant 1$ and set $\Omega=\widetilde{\Omega} / \varepsilon \omega$. For a stable cloud, the trapping potential is stronger than the inertial potential; hence we have $\Omega<1 / \varepsilon$. The energy can be rewritten as

$$
\begin{aligned}
E_{2 D}(u)= & \int \frac{1}{2}|\nabla u|^{2}+\frac{1}{2 \varepsilon^{2}}\left(x^{2}+\lambda^{2} y^{2}\right)|u|^{2}+\frac{1}{4 \varepsilon^{2}}|u|^{4} \\
& +\boldsymbol{\Omega} \cdot(i u, \boldsymbol{\nabla} u \times \mathbf{r}) .
\end{aligned}
$$

Due to the constraint $\int|u|^{2}=1$, we can add to $E_{2 D}$ any multiple of $\int|u|^{2}$ so that it is equivalent to minimizing

$$
\int|\boldsymbol{\nabla} u|^{2}+2 \boldsymbol{\Omega} \cdot(i u, \boldsymbol{\nabla} u \times \mathbf{r})+\frac{1}{2 \varepsilon^{2}}|u|^{4}-\frac{1}{\varepsilon^{2}} a(\mathbf{r})|u|^{2},
$$

where $a(\mathbf{r})=\alpha-\left(x^{2}+\lambda^{2} y^{2}\right)$ for some constant $\alpha$ to be determined. Let $\mathcal{D}$ be the ellipse $\{a>0\}=\left\{x^{2}+\lambda^{2} y^{2}<\alpha\right\}$. We impose the following constraint on $\alpha$ :

$$
\int_{\mathcal{D}} a(\mathbf{r})=1
$$

Indeed, as $\varepsilon$ tends to 0 , the minimizer will satisfy the constraint that $|u|^{2}$ will be close to $a$ so that the constraint will be satisfied automatically by $u$ if we impose Eq. (1.4). Note that $a$ is the Thomas-Fermi limit of $|u|^{2}$. Equation (1.4) leads to $\alpha^{2}=2 \lambda / \pi$. If $\lambda=1$, that is, $\omega_{x}=\omega_{y}$, then $\mathcal{D}$ is a disk, of radius $R_{0}$ with $R_{0}^{4}=2 / \pi$.

To study the problem analytically, it is reasonable to minimize the energy over the domain $\mathcal{D}$ with zero boundary data for $u$. Indeed, when $a \leqslant 0$, the energy is convex so that the minimizer $u$ goes to zero exponentially at infinity (see the numerical observation in [2] and the analysis of the behavior near the boundary of $\mathcal{D}$ as well as the decay at infinity of the order parameter in $[16,17])$. Denote by $H^{m}(\mathcal{D})$ the space of square integrable functions defined on the domain $\mathcal{D}$ that have square integrable derivatives up to order $m$, and by $H_{0}^{1}(\mathcal{D})$ the space of functions in $H^{1}(\mathcal{D})$ satisfying the zero boundary condition. Denote the norm $\left(\int_{\mathcal{D}}|v|^{2}\right)^{1 / 2}$ by $\|v\|$ for any square integrable function $v$. We then consider the problem

$$
\min E_{\varepsilon}(u) \quad \text { subject to } \quad u \in H_{0}^{1}(\mathcal{D}), \quad\|u\|=1,
$$

where

$$
E_{\varepsilon}(u)=\int_{\mathcal{D}}|\boldsymbol{\nabla} u|^{2}+2 \boldsymbol{\Omega} \cdot(i u, \boldsymbol{\nabla} u \times \mathbf{r})+\frac{1}{2 \varepsilon^{2}}\left[a(\mathbf{r})-|u|^{2}\right]^{2} .
$$

Note that the critical point $u$ of $E_{\varepsilon}$ is a solution of

$$
-\Delta u-2 i(\mathbf{\Omega} \times \mathbf{r}) \cdot \nabla u=\frac{1}{\varepsilon^{2}} u\left(a-|u|^{2}\right)+\mu_{\varepsilon} u \quad \text { in } \mathcal{D},
$$

with $u=0$ on $\partial \mathcal{D}$ and $\mu_{\varepsilon}$ the Lagrange multiplier. The specific choice of $\alpha$ in Eq. (1.4) will imply that the term $\mu_{\varepsilon} u$ is negligible compared to $a u / \varepsilon^{2}$.

We want to study the behavior of $\min E_{\varepsilon}(u)$ as $\varepsilon$ goes to 0 . In Sec. II, we compute an asymptotic expansion of the energy, and in Sec. III, the critical angular velocities of nucleation of vortices and the location of the vortices. In Sec. IV, we study the evolution in imaginary time and construct some numerical algorithms. In Sec. V, we present some computational results and the energy diagrams.

\section{ASYMPTOTIC EXPANSION OF THE ENERGY}

To study the behavior of the minimizer of the energy when $\varepsilon$ goes to zero, we observe that the form of the energy (1.5) is close to the Ginzburg-Landau functional studied in $[13,14]$, where the magnetic field has been replaced by a rotating term, and similar to [15] except for the trapping potential and the minimization over a constraint. The main idea is to decouple the energy into three terms: a part coming from the solution without vortices, a vortex contribution, and a term due to rotation. The estimate of the vortex contribution was developed in $[14,15,18,19]$.

\section{A. The solution without vortices}

We first consider solutions without vortices, that is, $u$ has no zero in the interior of $\mathcal{D}$. Given functions of the form $\eta$ $=f e^{i S}$, where $\eta$ is in $H_{0}^{1}(\mathcal{D})$ and $f$ is real and has no zero in the interior of $\mathcal{D}$, we consider minimizing $E_{\varepsilon}$ over such functions without imposing the constraint that the $L^{2}$ norm is 1 , that is, $f$ and $S$ minimize

$$
\begin{aligned}
\mathcal{E}_{\varepsilon}(f, S)= & \int_{\mathcal{D}}|\boldsymbol{\nabla} f|^{2}+\frac{1}{2 \varepsilon^{2}}\left(a-f^{2}\right)^{2}+\int f^{2}|\boldsymbol{\nabla} S-\mathbf{\Omega} \times \mathbf{r}|^{2} \\
& -f^{2} \Omega^{2} r^{2} .
\end{aligned}
$$

We have $f=0$ on $\partial \mathcal{D}$ and

$$
\begin{gathered}
-\Delta f+f \nabla S(\nabla S-2 \boldsymbol{\Omega} \times \mathbf{r})=\frac{1}{\varepsilon^{2}} f\left(a-f^{2}\right) \quad \text { in } \quad \mathcal{D}, \\
\operatorname{div}\left(f^{2}(\nabla S-\mathbf{\Omega} \times \mathbf{r})\right)=0 .
\end{gathered}
$$

From Eq. (2.3), there exists $\xi$ in $H^{2}(\mathcal{D}) \cap H_{0}^{1}(\mathcal{D})$ satisfying

$$
f^{2}(\nabla S-\mathbf{\Omega} \times \mathbf{r})=\Omega \nabla^{\perp} \xi,
$$


where $\boldsymbol{\nabla}^{\perp} \xi=\left(-\partial_{y} \xi, \partial_{x} \xi\right)$. So $\xi$ is the unique solution of

$$
\operatorname{div}\left(f^{-2} \nabla \xi\right)=0 \text { in } \mathcal{D}, \quad \xi=0 \text { on } \partial \mathcal{D} .
$$

Note that Eq. (2.4) is the equation for the velocity, but we prefer to write it as an orthogonal gradient for our later purposes of integration by parts. In the special case where $\mathcal{D}$ is a disk, the minimum of Eq. (2.1) is reached for $\boldsymbol{\nabla} S=\mathbf{0}$ but this is not the case if $\mathcal{D}$ is an ellipse and there is a nontrivial solution of Eq. (2.3).

\section{The case of the disk}

Assume that $\lambda=1$ so that $\mathcal{D}$ is a disk and $a(r)=R_{0}^{2}-r^{2}$. As discussed earlier, $\boldsymbol{\nabla} S=\mathbf{0}$ in this case so that the energy becomes

$$
\mathcal{E}_{\varepsilon}(f)=\int_{\mathcal{D}}|\nabla f|^{2}+\frac{1}{2 \varepsilon^{2}}\left[a(r)-f^{2}\right]^{2} .
$$

Let $\eta_{\varepsilon}$ be the minimizer of Eq. (2.6) in $H_{0}^{1}(\mathcal{D})$. Then, $\eta_{\varepsilon}$ has no vortex, is independent of $\Omega$, and satisfies

$$
\Delta \eta_{\varepsilon}=\frac{1}{\varepsilon^{2}} \eta_{\varepsilon}\left(\eta_{\varepsilon}^{2}-a\right) \text { in } \mathcal{D}, \quad \eta_{\varepsilon}=0 \text { on } \partial \mathcal{D} .
$$

When $\varepsilon$ tends to $0, \eta_{\varepsilon}^{2}$ is close to $a$ except on a boundary layer of size $\varepsilon^{2 / 3}$ close to $\partial \mathcal{D}$. More precisely, using sub- and supersolutions, one can verify that

$$
\sqrt{a(r)} \tanh \left[\delta(\sqrt{a(r)}]^{3}|\ln \varepsilon|\right) \eta_{\varepsilon} \leqslant \sqrt{a(r)}
$$

for $\left|R_{0}^{2}-r^{2}\right| \geqslant C \varepsilon^{1 / 3}$. In fact, one can construct a subsolution of the type above in any region $\left|R_{0}^{2}-r^{2}\right| \geqslant C \varepsilon^{\beta / 3}$ with $\beta$ $<2$. Then the value of $\delta$ is less than $c(2-\beta)$.

The boundary layer can be analyzed using the change of variables $x=\left(R_{0}-r\right) / \varepsilon^{2 / 3}$ and $v_{\varepsilon}(x)=\eta_{\varepsilon}(r) / \varepsilon^{2 / 3} \cdot v_{\varepsilon}$ satisfies the Painlevé equation $v^{\prime \prime}=v\left(v^{2}-2 R_{0} x\right)$ with $v(0)=0$ and $v(x) \simeq 2 R_{0} x$ for $x$ large. The boundary behavior has already been studied in [17] and [16] using matched asymptotics.

The energy of $\eta_{\varepsilon}$ can also be estimated by a test function equal to $\sqrt{a}$ except on the boundary layer to get

$$
E_{\varepsilon}\left(\eta_{\varepsilon}\right) \leqslant \frac{2 \pi}{3}|\ln \varepsilon|[1+o(1)] .
$$

\section{The case of an ellipse}

As discussed before, the minimum $\eta_{\varepsilon}=f_{\varepsilon} e^{i S_{\varepsilon}}$ of Eq. (2.1) has a nontrivial phase. $f_{\varepsilon}^{2}$ tends to $a$ in every compact subset of $\mathcal{D}$ and the function $\xi_{\varepsilon}$ given by Eq. (2.4) or (2.5) tends to the unique solution $\xi$ of

$$
\operatorname{div}\left(\frac{1}{a} \nabla \xi\right)=0 \text { in } \mathcal{D}, \quad \xi=0 \text { on } \partial \mathcal{D} .
$$

One can easily get that $\xi(x, y)=-a^{2}(x, y) /\left(2+2 \lambda^{2}\right)$. Using Eq. (2.4), we can define $S_{0}$, the limit of $S_{\varepsilon}$, to be the solution of $a\left(\boldsymbol{\nabla} S_{0}-\boldsymbol{\Omega} \times \mathbf{r}\right)=\Omega \nabla^{\perp} \xi$ with zero value at the origin. We have $S_{0}=C \Omega x y$ with $C=\left(\lambda^{2}-1\right) /\left(\lambda^{2}+1\right)$. We see that $S_{0}$ cancels when $\lambda=1$, that is, in the case of the disk. This computation is consistent with the one in [10], although it is derived in a different way.

\section{B. Decoupling the energy}

Let $\eta_{\varepsilon}=f_{\varepsilon} e^{i S_{\varepsilon}}$ be the vortex-free minimizer of $E_{\varepsilon}$ discussed previously without imposing the constraint on the norm of $u$. Let $u_{\varepsilon}$ be a minimizer of $E_{\varepsilon}$ under the constraint $\int_{\mathcal{D}}|u|^{2}=1$ and let $v_{\varepsilon}=u_{\varepsilon} / \eta_{\varepsilon}$. Since $\eta_{\varepsilon}$ satisfies the GrossPitaevskii equations (2.2) and (2.3), we have

$$
\begin{gathered}
\int_{\mathcal{D}}\left(|v|^{2}-1\right)\left(-\frac{1}{2} \Delta f_{\varepsilon}^{2}-\frac{1}{\varepsilon^{2}} f_{\varepsilon}^{2}\left(a-f_{\varepsilon}^{2}\right)+\left|\nabla f_{\varepsilon} e^{i S_{\varepsilon}}\right|^{2}\right. \\
\left.-2 f_{\varepsilon}^{2}\left(\nabla S_{\varepsilon} \cdot \mathbf{\Omega} \times \mathbf{r}\right)\right)=0 .
\end{gathered}
$$

This implies that the energy $E_{\varepsilon}\left(u_{\varepsilon}\right)$ decouples as follows:

$$
\begin{aligned}
E_{\varepsilon}\left(u_{\varepsilon}\right)= & E_{\varepsilon}\left(\eta_{\varepsilon}\right)+G_{\varepsilon}\left(v_{\varepsilon}\right) \\
& +2 \int_{\mathcal{D}}\left|\eta_{\varepsilon}\right|^{2}\left(\nabla S_{\varepsilon}-\boldsymbol{\Omega} \times \mathbf{r}\right) \cdot\left(i v_{\varepsilon}, \nabla v_{\varepsilon}\right),
\end{aligned}
$$

where

$$
G_{\varepsilon}\left(v_{\varepsilon}\right)=\int_{\mathcal{D}}\left|\eta_{\varepsilon}\right|^{2}\left|\nabla v_{\varepsilon}\right|^{2}+\frac{\left|\eta_{\varepsilon}\right|^{4}}{2 \varepsilon^{2}}\left(1-\left|v_{\varepsilon}\right|^{2}\right)^{2}
$$

This decoupling was used in [15] in the case of a disk where $\boldsymbol{\nabla} S_{\varepsilon}=\mathbf{0}$.

\section{Estimate of the energy}

We now estimate the terms in Eq. (2.9). The first term $E_{\varepsilon}\left(\eta_{\varepsilon}\right)$ is a constant depending only on $\varepsilon$, and not on the solution type, that is, with or without vortices. The second term gives a contribution coming from the vortices and the third term is due to the vortices and rotation.

We use the analysis of vortices developed in [18] and later in $[14,15,19]$. Let $\mathcal{D}_{\varepsilon}^{\prime}=\mathcal{D} \backslash\left\{x, \operatorname{dist}(x, \partial \mathcal{D}) \leqslant \varepsilon^{\beta}\right\}$, with $\beta<1$. Then in $\mathcal{D}_{\varepsilon}^{\prime}$ it is possible to define vortices for $v_{\varepsilon}$ in the following way. There exist balls $B_{i}=B\left(p_{i}, \varepsilon^{\beta^{\prime}}\right)$ where $p_{i}$ are points in $\mathcal{D}_{\varepsilon}^{\prime}$ at mutual distance bigger than $8 \varepsilon^{\beta^{\prime}}$ and $\beta^{\prime}$ $>\beta$, such that $\left|v_{\varepsilon}\right| \geqslant 1 / 2$ in $\mathcal{D}_{\varepsilon}^{\prime} \backslash \cup_{i} B_{i}$. Moreover, the degree $d_{i}=\operatorname{deg}\left(v_{\varepsilon} /\left|v_{\varepsilon}\right|, \partial B_{i}\right)$ is not zero and there is an estimate of the energy of $v_{\varepsilon}$ in each ball $B_{i}$. This analysis means that vortices are in fact defined in the balls where $v_{\varepsilon}$ is less than $1 / 2$ and has a nonzero degree. This allows us to compute $G_{\varepsilon}\left(v_{\varepsilon}\right)$ for which only the gradient term in the vortex balls will give a contribution: each vortex gives a contribution in the amount of $2 \pi|\ln \varepsilon|$ due to its degree and a contribution of lower order that comes from the interaction with the other vortices. Moreover, $\left|\eta_{\varepsilon}\right|^{2}$ is almost $a$ : 


$$
\begin{aligned}
G_{\varepsilon}(v)= & 2 \pi|\ln \varepsilon| \sum_{i}\left|d_{i}\right| a\left(p_{i}\right) \\
& -2 \pi \sum_{i \neq j} d_{i} d_{j} \sqrt{a\left(p_{i}\right) a\left(p_{j}\right)} \ln \left|p_{i}-p_{j}\right|+O(1) .
\end{aligned}
$$

In order to estimate the third term in Eq. (2.9) we let $X_{\varepsilon}$ be the solution of $a(\mathbf{r})\left(\boldsymbol{\nabla} S_{0}-\boldsymbol{\Omega} \times \mathbf{r}\right)=\Omega \boldsymbol{\nabla}^{\perp} X_{\varepsilon}$ that is zero on the boundary of $\mathcal{D}_{\varepsilon}^{\prime}$. That is, $X_{\varepsilon}$ solves Eq. (2.8) but with zero boundary data on $\partial \mathcal{D}_{\varepsilon}^{\prime}$ instead of $\partial \mathcal{D}$. Hence $X_{\varepsilon}$ converges to $\xi$ the solution of Eq. (2.8). An integration by parts on the last term of Eq. (2.9) using the definition of $X_{\varepsilon}$ and the definition of the degree of $v_{\varepsilon}$ on vortex balls and the fact that the higher-order term comes from an integration on the vortex balls yields

$$
\begin{aligned}
\int_{\mathcal{D}}\left|\eta_{\varepsilon}\right|^{2}\left(\nabla S_{\varepsilon}-\boldsymbol{\Omega} \times \mathbf{r}\right) \cdot(i v, \nabla v) \\
\quad=\int_{\mathcal{D}_{\varepsilon}^{\prime} \backslash \cup_{i} B_{i}} \boldsymbol{\Omega} \cdot\left(i v, d X_{\varepsilon} \times \boldsymbol{\nabla} v\right)[1+o(1)] \\
=\sum_{i} 2 \pi \Omega d_{i} X_{\varepsilon}\left(p_{i}\right)[1+o(1)] \\
=\sum_{i} \frac{-\pi \Omega d_{i}}{1+\lambda^{2}}\left(\alpha-\left|x_{i}\right|^{2}-\lambda^{2}\left|y_{i}\right|^{2}\right)^{2}[1+o(1)] .
\end{aligned}
$$

Finally, one can derive from Eqs. (2.9)-(2.11) an asymptotic expansion of the energy for a solution with vortices:

$$
\begin{aligned}
E_{\varepsilon}\left(u_{\varepsilon}\right)-E_{\varepsilon}\left(\eta_{\varepsilon}\right) \simeq & 2 \pi|\ln \varepsilon| \sum_{i}\left|d_{i}\right| a\left(p_{i}\right) \\
& -\frac{2 \pi \Omega}{1+\lambda^{2}} \sum_{i} d_{i}\left(\alpha-\left|x_{i}\right|^{2}-\lambda^{2}\left|y_{i}\right|^{2}\right)^{2} \\
& -2 \pi \sum_{i \neq j} d_{i} d_{j} \sqrt{a\left(p_{i}\right) a\left(p_{j}\right)} \ln \left|p_{i}-p_{j}\right| .
\end{aligned}
$$

Note that the minimal energy for solutions without vortices in $\mathcal{D}_{\varepsilon}^{\prime}$ is $E_{\varepsilon}\left(\eta_{\varepsilon}\right)+O(\varepsilon|\ln \varepsilon|)$ : it is not exactly $E_{\varepsilon}\left(\eta_{\varepsilon}\right)$ since $\eta_{\varepsilon}$ is a minimizer without the constraint $\left\|\eta_{\varepsilon}\right\|=1$, but it almost equals $E_{\varepsilon}\left(\eta_{\varepsilon}\right)$ since $\int_{\mathcal{D}} a=1$ and $\left|\eta_{\varepsilon}\right|^{2}$ approaches $a$ asymptotically. The above estimate illustrates that, physically speaking, the energy of solutions having $n$ vortices is approximately given by the energy of the vortexless solution and the sum of the self-energy of individual vortices and the interaction energy of vortex pairs. This fact was also observed in [2] using a different approach.

\section{CRITICAL ANGULAR VELOCITIES}

\section{A. Critical angular velocity for the existence of one vortex}

Let $u_{\varepsilon}$ be a minimizer of $(P)$ with one vortex at a point $p$ in $\mathcal{D}$ with coordinates $(x, y)$ and let $\Delta E_{\varepsilon}$ be the difference between $E_{\varepsilon}\left(u_{\varepsilon}\right)$ and the energy of a solution without vortex $\left[E_{\varepsilon}\left(\eta_{\varepsilon}\right)+O(\varepsilon|\ln \varepsilon|)\right]$ :

$$
\begin{aligned}
\Delta E_{\varepsilon}= & 2 \pi\left(\alpha-x^{2}-\lambda^{2} y^{2}\right)\left(|\ln \varepsilon|-\frac{\Omega}{1+\lambda^{2}}\left(\alpha-x^{2}-\lambda^{2} y^{2}\right)\right) \\
& \times[1+o(1)] .
\end{aligned}
$$

This expression was obtained by Svidzinsky and Fetter [10] using a different method. The form of $\Delta E_{\varepsilon}$ allows the computation of two critical angular velocities $\Omega_{s}$ and $\Omega_{1}$ for the existence of vortices: $\Omega_{s}$ is the velocity for which the solution with one vortex starts to be locally stable and $\Omega_{1}$ that for which it starts to be globally stable. For $\Omega<\Omega_{s}, \Delta E_{\varepsilon}$ is a decreasing function of $|p|$, the position of the vortex; $|p|$ $=0$ is a local maximum of $\Delta E_{\varepsilon}$. For $\Omega_{s}<\Omega<\Omega_{1},|p|=0$ is a local minimum for $\Delta E_{\varepsilon}$. Note that $\Delta E_{\varepsilon}(p \in \partial D)=0$ and $\Delta E_{\varepsilon}(|p|=0)>0$.

For $\Omega>\Omega_{1},|p|=0$ is the global minimum for $\Delta E_{\varepsilon}$. We thus have

$$
\begin{aligned}
& \Omega_{s}=\frac{1+\lambda^{2}}{2 \alpha}|\ln \varepsilon|=\frac{1+\lambda^{2}}{4 \sqrt{\lambda}} \sqrt{2 \pi}|\ln \varepsilon|, \\
& \Omega_{1}=\frac{1+\lambda^{2}}{\alpha}|\ln \varepsilon|=\frac{1+\lambda^{2}}{2 \sqrt{\lambda}} \sqrt{2 \pi}|\ln \varepsilon|,
\end{aligned}
$$

that is,

$$
\begin{aligned}
& \widetilde{\Omega}_{s}=\frac{1+\lambda^{2}}{4 \sqrt{\lambda}} \omega \sqrt{\frac{\pi \hbar^{2}}{N g m}} \ln \left(\frac{N g m}{\hbar^{2}}\right)^{1 / 2}, \\
& \widetilde{\Omega}_{1}=\frac{1+\lambda^{2}}{2 \sqrt{\lambda}} \omega \sqrt{\frac{\pi \hbar^{2}}{N g m}} \ln \left(\frac{N g m}{\hbar^{2}}\right)^{1 / 2} .
\end{aligned}
$$

Note that Castin and Dum [2] for the case $\lambda=1$ find $\widetilde{\Omega}_{1}$ $=\omega \sqrt{\left(\pi \hbar^{2}\right) /(N g m)} \ln \left[(C / \sqrt{\pi})(N g m) / \hbar^{2}\right]^{1 / 2}$ with $C \simeq 1.8$, and hence $C / \sqrt{\pi} \simeq 1$, which gives a value of $\widetilde{\Omega}_{1}$ very close to ours. They also have $\Omega_{1}=2 \Omega_{s}$ for the case $\lambda=1$. Increases in anisotropy yield higher $\widetilde{\Omega}_{1}$ as already noticed in [4] and experimentally confirmed in $[8,9]$, but as $\lambda$ tends to infinity $\Omega_{1}$ becomes bigger than $1 / \varepsilon$ so that vortices cannot be stabilized.

It can be proved that there exists $k_{\varepsilon}$ that tends to zero with $\varepsilon$ such that for $\Omega<\Omega_{1}-k_{\varepsilon}$ the minimizer of $E_{\varepsilon}$ has no vortex and for $\Omega>\Omega_{1}+k_{\varepsilon}$ there exists a minimizer with a vortex. Such a property rigorously justifies the physical meaning of $\Omega_{1}$ as the critical angular velocity for the existence of a minimizer with one vortex. Moreover, for $\Omega_{1}+k_{\varepsilon}<\Omega<\Omega_{1}$ $+O(1)$, any minimizer has one vortex of degree 1 tending to the origin. The proof consists in constructing a test function 
with a vortex at the origin and computing the energy of this test function. This yields an upper bound for the energy. The lower bound relies on estimates for $G_{\varepsilon}(v)$ from [18] and $[14,15]$.

\section{B. Critical angular velocity for $\boldsymbol{n}$ vortices}

Similarly, one can compute $\Omega_{n}$, the critical angular velocity for the existence of $n$ vortices. For this purpose, one can prove that as $\varepsilon$ goes to 0 the vortices tend to the origin and they are all singly quantized vortices, that is $d_{i}=1$. The proof goes as in $[14,15]$ : to show it, one may define a test function that consists in putting the $n$ vortices on a polygon centered at the origin of size $1 / \sqrt{\Omega}$ in $x$ and $1 / \lambda \sqrt{\Omega}$ in $y$, and check that this has a lower energy than any configuration with multiple vortices. Using a special ansatz, it has been demonstrated in [2] that multiply quantized vortices are unstable. In fact, it is a physical signature of harmonic trapping potentials that the minimizers have only single vortices. When other trapping potentials are used, it is possible to have minimizers with multiply quantized vortices $\left(d_{i}>1\right)$; see, for example, the discussion in [20] for quartic potentials.

We let $\tilde{p}_{i}$ with coordinates $\left(\tilde{x}_{i}, \tilde{y}_{i}\right)$ be such that $\tilde{x}_{i}$ $=x_{i} \sqrt{\Omega}$ and $\tilde{y}_{i}=\lambda y_{i} \sqrt{\Omega}$. This allows us to estimate the energy of a solution with $n$ vortices centered at $\tilde{p}_{i}$ from Eqs. (2.9)-(2.11):

$$
\begin{aligned}
E_{\varepsilon}(u)= & E_{\varepsilon}\left(\eta_{\varepsilon}\right)+2 \pi n \alpha\left(|\ln \varepsilon|-\frac{1}{1+\lambda^{2}} \Omega \alpha\right) \\
& +\pi\left(n^{2}-n\right) \alpha \ln \Omega+w\left(\tilde{p}_{1}, \ldots, \tilde{p}_{n}\right)+C_{n}+o(1)
\end{aligned}
$$

where $C_{n}$ is a constant that depends on $n$ and $\lambda$ and

$$
\begin{aligned}
w\left(\tilde{p}_{1}, \ldots, \tilde{p}_{n}\right)= & -2 \pi \alpha \sum_{i \neq j} \ln \left(\left|\tilde{x}_{i}-\tilde{x}_{j}\right|^{2}+\frac{\left|\tilde{y}_{i}-\tilde{y}_{j}\right|^{2}}{\lambda^{2}}\right) \\
& +2 \pi \alpha \sum_{i}\left(\tilde{x}_{i}^{2}+\tilde{y}_{i}^{2}\right)\left(\frac{2}{1+\lambda^{2}}-\frac{|\ln \varepsilon|}{\Omega \alpha}\right) .
\end{aligned}
$$

The first term in Eq. (3.3) gives the repulsive interaction between the vortices and the second term illustrates the competition between the restoring and centrifugal forces $[8,9]$.

Recall that $\alpha^{2}=2 \lambda / \pi$. For fixed $\lambda, w$ is of order 1, and hence is of lower order than the previous terms. Then the critical angular velocity for the existence of $n$ vortices can be computed from Eq. (3.2),

$$
\begin{aligned}
\Omega_{n}= & \left(1+\lambda^{2}\right) \sqrt{\frac{\pi}{2 \lambda}}[\ln \varepsilon \mid \\
& \left.+\frac{(n-1)}{2} \ln \left(\left(1+\lambda^{2}\right) \sqrt{\frac{\pi}{2 \lambda}}|\ln \varepsilon|\right)\right],
\end{aligned}
$$

and the critical angular velocity in the original parameters is $\widetilde{\Omega}_{n}$,

$$
\begin{aligned}
\widetilde{\Omega}_{n}= & \frac{\left(1+\lambda^{2}\right)}{2} \omega \sqrt{\frac{\pi \hbar^{2}}{N g m \lambda}}\left\{\ln \left(\frac{N g m}{\hbar^{2}}\right)^{1 / 2}\right. \\
& \left.+\frac{(n-1)}{2} \ln \left[\frac{1+\lambda^{2}}{2 \sqrt{\lambda}} \sqrt{2 \pi} \ln \left(\frac{N g m}{\hbar^{2}}\right)^{1 / 2}\right]\right\} .
\end{aligned}
$$

Clearly, this is in agreement with the experimental observation that for larger $\lambda$, that is, more intense anisotropy, the critical angular velocities have wider bands $[8,9]$.

\section{Location of vortices}

Once $\Omega$ is close to $\Omega_{n}$, the location of the vortices is characterized by the configuration of points $\left\{\tilde{p}_{i}\right\}$ that minimizes the function $w$ given by Eq. (3.3). In nondimensionalized variables, the points are given by $R p_{i} / \sqrt{2 \pi|\ln \varepsilon|}$. For convenience, we define

$$
\rho=\frac{2}{\left(1+\lambda^{2}\right)}-\frac{|\ln \varepsilon|}{\Omega \alpha} .
$$

Note that, given the value of $\Omega_{n}$ in Eq. (3.4), to leading order, $\rho$ is equal to $\sqrt{2 \pi / \lambda} /\left(1+\lambda^{2}\right)$. We use the values of $\alpha$ and $\rho$ to get a simplified expression for $w$ :

$$
\begin{aligned}
w\left(\tilde{p}_{1}, \ldots, \tilde{p}_{n}\right)= & -\sqrt{8 \pi \lambda}\left[\rho \sum_{i}\left(\tilde{x}_{i}^{2}+\tilde{y}_{i}^{2}\right)\right. \\
& \left.+\sum_{i \neq j} \ln \left(\left|\tilde{x}_{i}-\tilde{x}_{j}\right|^{2}+\frac{\left|\tilde{y}_{i}-\tilde{y}_{j}\right|^{2}}{\lambda^{2}}\right)\right] .
\end{aligned}
$$

The critical points of $w$, and thus the vortex positions, satisfy

$$
\begin{aligned}
& \rho \tilde{x}_{i}=\sum_{j \neq i} \frac{\lambda^{2}\left(\tilde{x}_{i}-\tilde{x}_{j}\right)}{\lambda^{2}\left|\tilde{x}_{i}-\tilde{x}_{j}\right|^{2}+\left|\tilde{y}_{i}-\tilde{y}_{j}\right|^{2}}, \\
& \rho \tilde{y}_{i}=\sum_{j \neq i} \frac{\tilde{y}_{i}-\tilde{y}_{j}}{\lambda^{2}\left|\tilde{x}_{i}-\tilde{x}_{j}\right|^{2}+\left|\tilde{y}_{i}-\tilde{y}_{j}\right|^{2}} .
\end{aligned}
$$

An immediate observation is that

$$
\sum_{i} \tilde{x}_{i}=\sum_{i} \tilde{y}_{i}=0
$$

By multiplying the equations by $\tilde{x}_{i}$ and $\tilde{y}_{i}$, respectively, and adding the results together, one can obtain

$$
\sum_{i}\left(\tilde{x}_{i}^{2}+\tilde{y}_{i}^{2}\right)=n(n-1) /(2 \rho) .
$$

Similarly, multiplying the equations by $\tilde{y}_{i}$ and $-\lambda^{2} \tilde{x}_{i}$, respectively, and adding the results together, one gets

$$
\rho\left(1-\lambda^{2}\right) \sum_{i} \tilde{x}_{i} \tilde{y}_{i}=0 .
$$


Unlike Eqs. (3.8) and (3.9) where the dependence on $\lambda$ is implicit, Eq. (3.10) leads to the property

$$
\sum_{i} \tilde{x}_{i} \tilde{y}_{i}=0, \quad \text { for } \lambda \neq 1
$$

The above observations lead to more precise predictions of the location of vortices.

For instance, in the case $n=2$ we get $\tilde{x}_{1}=-\tilde{x}_{2}$ and $\tilde{y}_{1}$ $=-\tilde{y}_{2}$. For $\lambda=1$, we have an infinite set of solutions consisting in two points on the circle $\tilde{x}_{i}^{2}+\tilde{y}_{i}^{2}=1 / \rho$, symmetric with respect to the origin. For $\lambda \neq 1$, Eq. (3.11) leads to $\tilde{x}_{i} \tilde{y}_{i}=0$ for $i=1,2$, and we have a pair of solutions with either $\tilde{x}_{i}=0, \tilde{y}_{i}= \pm \sqrt{1 / 2 \rho}$, or $\tilde{y}_{i}=0, \tilde{x}_{i}= \pm \sqrt{1 / 2 \rho}$. Checking the corresponding values of $w$, we get that for $\lambda \neq 1$ the minimizer of $w$ corresponds to having both vortices staying on the long axis of the ellipse in the original scaling (that is, on the $x$ axis if $\lambda>1$ and on the $y$ axis if $\lambda<1$ ). This estimate of the location is in agreement with the numerical solutions given later.

For the case $n=3$, we also get that the three vortices are on the long axis of the ellipse: one centered at the origin whereas the other pair stays symmetrically on the long axis with $\tilde{x}_{i}= \pm \sqrt{3 / 2 \rho}$ if $\lambda>1$. Similar discussions can be carried out for other values of $n$. In general, for the case of $n$ vortices, we let $R_{1}^{2}=n(n-1) / 2 \rho$ and $\hat{x}_{i}=R_{1} \tilde{x}_{i}, \hat{y}_{i}=R_{1} \tilde{y}_{i}$. It follows from Eq. (3.9) that the points $\left\{\tilde{p}_{i}\right\}$ are localized by the minimum of

$$
-\sum_{i \neq j} \ln \left(\left|\hat{x}_{i}-\hat{x}_{j}\right|^{2}+\frac{\left|\hat{y}_{i}-\hat{y}_{j}\right|^{2}}{\lambda^{2}}\right)
$$

under the constraint $\Sigma_{i} \hat{x}_{i}^{2}+\hat{y}_{i}^{2}=1$. It is reasonable to expect that this formula will lead to a vortex array as computed in [21] and observed in [9] containing a moderate number of vortices. Note that the minimizer $\left\{\hat{p}_{i}=R_{1} \tilde{p}_{i}\right\}$ has no explicit dependence on $\Omega$; thus, for a given $\lambda$, we expect the vortex configuration to be of similar structure for values of $\Omega$ close to $\Omega_{n}$. On the other hand, for a given $n$ and $\Omega$, we expect that for sufficiently large $\lambda$, that is, for highly anisotropic traps, the minimizer of $w$ to be given by collinear solutions with vortices all located on the long axis of the elliptical trap. Our characterization of the location of the vortices is consistent with the numerical results given later.

\section{EVOLUTION EQUATION AND NUMERICAL SCHEMES}

To numerically compute the energy minimizers of Eq. (1.5), we notice that the energy in Eq. (1.5) can be rewritten as

$$
\int_{\mathcal{D}}\left\{|(\boldsymbol{\nabla}-i \mathbf{A}) u|^{2}+\frac{1}{2 \varepsilon^{2}}\left[a_{\varepsilon}(\mathbf{r})-|u|^{2}\right]^{2}\right\}+c_{\varepsilon}
$$

where $a_{\varepsilon}(\mathbf{r})=a(\mathbf{r})-\varepsilon^{2} \Omega^{2} r^{2}$,

$$
\mathbf{A}=\left(\begin{array}{c}
y \\
-x
\end{array}\right) \mathbf{\Omega}, \quad \text { and } \quad c_{\varepsilon}=\int_{\mathcal{D}}\left\{\frac{1}{2 \varepsilon^{2}}\left[a^{2}(\mathbf{r})-a_{\varepsilon}^{2}(\mathbf{r})\right]\right\} .
$$

The above formulation of the energy has a striking similarity with the high- $\kappa$ high-field Ginzburg-Landau energy [22] with a variable coefficient [23].

\section{A. Evolution in imaginary time}

To numerically compute the minimizers of Eq. (1.5), we consider the time-dependent equation in imaginary time:

$$
\frac{\partial u}{\partial t}-(\boldsymbol{\nabla}-i \mathbf{A})^{2} u+\frac{1}{\varepsilon^{2}}|u|^{2} u-\frac{a_{\varepsilon}(\mathbf{r})}{\varepsilon^{2}} u=\mu_{\varepsilon}(u) u
$$

in $\mathcal{D}$ with initial condition $u(\mathbf{r}, 0)=u_{0}(\mathbf{r})$ in $\mathcal{D}$ and boundary condition $u=0$ on $\partial \mathcal{D}$. Here, $\mu_{\varepsilon}(u)$ denotes the Lagrange multiplier. Assume that $u_{0}$ satisfies the constraint $\left\|u_{0}\right\|=1$. Then, by taking

$$
\mu_{\varepsilon}(u)=\int_{\mathcal{D}}\left\{|(\boldsymbol{\nabla}-i \mathbf{A}) u|^{2}+\frac{1}{\varepsilon^{2}}|u|^{4}-\frac{a_{\varepsilon}(\mathbf{r})}{\varepsilon^{2}}|u|^{2}\right\} d \mathcal{D},
$$

we get

$$
\frac{d}{d t}\left(\int_{\mathcal{D}}|u|^{2} d \mathcal{D}-1\right)-\mu_{\varepsilon}(u)\left(\int_{\mathcal{D}}|u|^{2} d \mathcal{D}-1\right)=0 .
$$

Thus, the constraint $\int_{\mathcal{D}}|u|^{2}=1$ is ensured at all times. Moreover, using $\left(u, u_{t}\right)=0$, we get the energy estimate

$$
\frac{1}{2} \frac{d}{d t} \mathcal{E}_{\varepsilon}(u)+\left\|\frac{\partial u}{\partial t}\right\|^{2}=0
$$

Thus, we easily get that for any $(0, T)$, if $u_{0} \in H_{0}^{1}(\mathcal{D})$ and $\left\|u_{0}\right\|=1$, there exists a unique strong solution $u$ of Eq. (4.2) satisfying the constraint $\|u\|=1$. Using an argument similar to that in [24,25], we may also get that as $t \rightarrow \infty u$ approaches a steady state solution which is a critical point of the energy. Well-posedness for $L^{2}$ initial data may also be obtained.

\section{B. Numerical schemes}

There are various ways to solve the time-dependent Gross-Pitaevskii equations; see, for example, [2] or [4]. We take advantage of the striking similarity with the high- $\kappa$ high-field time-dependent Ginzburg-Landau equations [26], and adapt a code developed in $[23,26,27]$. Spatially, we use a standard finite element approximation; see $[23,27]$ for details. Here, we focus on the time discretization and the treatment of constraints. It has been observed that there are some steady states exhibiting metastability; thus it is important to get asymptotically stable schemes for large times, which in general require the use of implicit schemes with no limitations on the time step size.

Let $\left\{u_{n}\right\}$ be approximate solutions of $\left\{u\left(t_{n}\right)\right\}$ at discrete time $\left\{t_{n}\right\}$ with time step $\Delta t_{n}=t_{n}-t_{n-1}$. We discuss two time-discretization schemes and also some results of numerical experiments. 


\section{A first order backward Euler in time discretization}

Given $u_{n-1}$, we first solve for $u^{*}$ :

$$
\begin{aligned}
& \frac{u^{*}-u_{n-1}}{\Delta t_{n}}-(\boldsymbol{\nabla}-i \mathbf{A})^{2} u^{*}-\mu\left(u_{n-1}\right) u^{*}+\frac{1}{\varepsilon^{2}}\left|u^{*}\right|^{2} u^{*} \\
& -\frac{1}{\varepsilon^{2}} a_{\varepsilon} u^{*}=0 .
\end{aligned}
$$

Then, we apply the projection $u_{n}=u^{*} /\left\|u^{*}\right\|$. Both the backward Euler step and the projection step give only first order in time accuracy.

\section{A norm-preserving, energy-decreasing second order scheme}

For any $u, v$ and their complex conjugates $\bar{u}, \bar{v}$, we let $f(u, v)=\left(|u|^{2}+|v|^{2}\right)(u+v) / 2$, which satisfies $f(u, v)(\bar{u}$ $-\bar{v})=\left(|u|^{4}-|v|^{4}\right) / 2$. Given $u_{n-1}$, we first solve for $u^{*}$ :

$$
\begin{aligned}
& \frac{2\left(u^{*}-u_{n-1}\right)}{\Delta t_{n}}-(\boldsymbol{\nabla}-i \mathbf{A})^{2} u^{*}-\nu\left(u^{*}\right) u^{*} \\
& +\frac{1}{\varepsilon^{2}} f\left(2 u^{*}-u_{n-1}, u_{n-1}\right)-\frac{1}{\varepsilon^{2}} a_{\varepsilon} u^{*}=0,
\end{aligned}
$$

where $\nu\left(u^{*}\right)$ is given by

$$
\begin{aligned}
\nu\left(u^{*}\right) \int_{\mathcal{D}}\left|u^{*}\right|^{2}= & \int_{\mathcal{D}}\left\{\left|(\boldsymbol{\nabla}-i \mathbf{A}) u^{*}\right|^{2}\right\} \\
& +\int\left\{\frac{1}{\varepsilon^{2}} f\left(2 u^{*}-u_{n-1}, u_{n-1}\right) \bar{u}^{*}\right. \\
& \left.-\frac{a_{\varepsilon}}{\varepsilon^{2}}\left|u^{*}\right|^{2}\right\} .
\end{aligned}
$$

Then we let $u_{n}=2 u^{*}-u_{n-1}$. Taking the inner product of Eq. (4.4) with $u^{*}$, we get $\left(u^{*}-u_{n-1}, u^{*}\right)=0$, which leads to $\left\|u_{n}\right\|^{2}=\left\|u_{n-1}\right\|^{2}$. That is, the norm is preserved at each time step. Taking the inner product of Eq. (4.4) with $u^{*}-u_{n-1}$, it is easy to get

$$
2 \frac{\left\|u_{n}-u_{n-1}\right\|^{2}}{\Delta t_{n}}+\mathcal{E}_{\varepsilon}\left(u_{n}\right)-\mathcal{E}_{\varepsilon}\left(u_{n-1}\right)=0 .
$$

Thus, during the discrete time evolution, the energy decreases. This discrete scheme is second order in time and unconditionally stable. It also preserves some essential features of the continuous dynamic system, making it suitable for long time integration and for studies of metastabilities of the solutions.

\section{Description of the numerical experiments}

We have used the above schemes to calculate various numerical solutions for the parameter values $\varepsilon=0.02, \lambda=1$, and $\lambda=1.5$. The spatial finite element space is taken to be $C^{0}$ piecewise quadratic elements on triangular meshes. As we
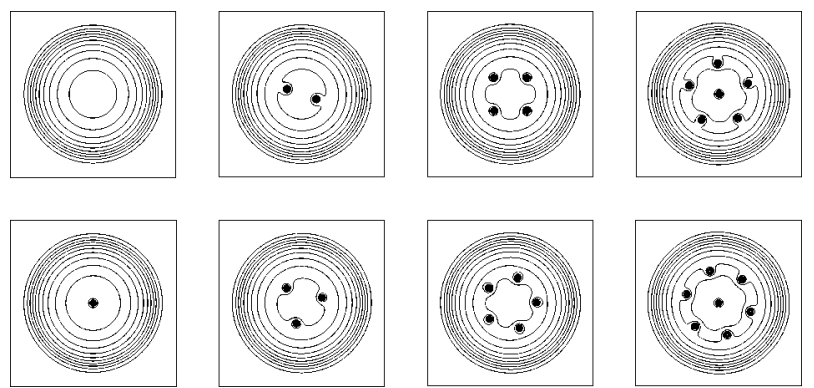

FIG. 1. Contour plots of $|u|$ for $\Omega=0$ (first column), 15 (second column) 17.5 (third column), and 20 (fourth column).

are mostly interested in the minimizers of the GrossPitaevskii energy, the time evolution is employed as a means of marching to the steady state solutions. For this reason, we have used variable time steps in order to accelerate the convergence in time. The nonlinear systems are solved by a Newton-like method at each step. Although such a method is computationally costly per step, this drawback is offset by its unconditional asymptotic stability for marching to the steady state. We have also computed the solutions using refined meshes to ascertain the numerical convergence.

To obtain solutions for various velocities, we have used a number of different initial conditions. For example, we have used $\left|u_{0}(\mathbf{r})\right|^{2}=a(\mathbf{r})$ for $\mathbf{r} \in \mathcal{D}$, which serves as a good approximation to the steady state solution, especially in the case of vortex-free solutions. We note that for large values of $\Omega$ this choice of initial condition can also lead to steady state solutions with multiple vortices. Detailed solution branches are described in the next section. In addition, we have also used other initial conditions that manually plant vortices in the domain in order to find different solution branches. Finally, a continuation in the parameter $\Omega$ has often been employed to follow a particular solution branch and to compute the bifurcation diagrams. The continuation procedure also provides a test for the local stability of the numerical solutions: when one branch becomes unstable, the solution jumps onto a different branch.

\section{NUMERICAL RESULTS AND BIFURCATION DIAGRAMS}

We now present some pictures of numerical solutions and discuss the various solution branches.

\section{A. Description of solutions}

\section{The case of a disk}

For any $\Omega$, there is a vortex-free solution, which is close to $a(x, y)$ except near the boundary. For $\Omega=0$, in addition to
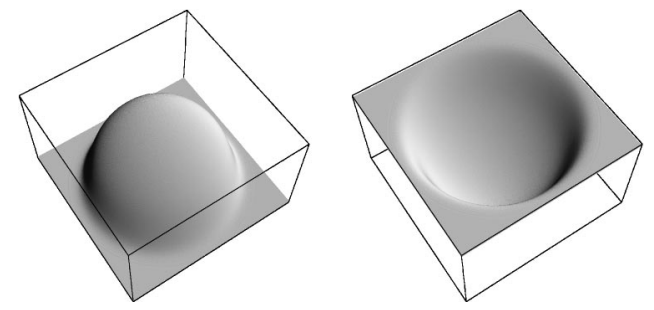

FIG. 2. Surface plots of $|u|$ for solutions at $\Omega=0$. 

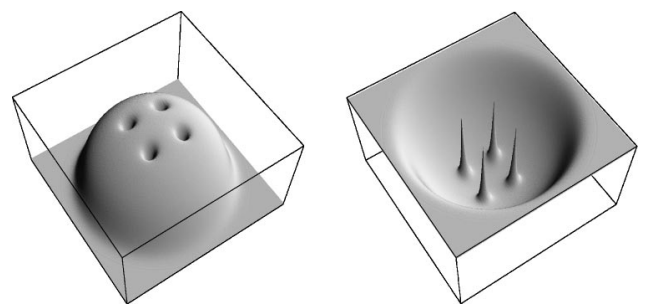

FIG. 3. Surface plots of $|u|$ for solutions at $\Omega=20$.

this vortex-free solution, there is also a one-vortex solution, as illustrated in the first column of Fig. 1. The square domain showing in each picture is of size 2.2 in both directions.

For larger $\Omega$, solutions with multiple vortices are shown in the other columns of Fig. 1. For instance, solutions with two and three vortices for $\Omega=15$ are shown in the second column, solutions with four and five vortices for $\Omega=17.5$ are shown in the third column, and solutions with six and seven vortices for $\Omega=20$ are shown in the last column. The parameter values for which the single-vortex and multivortex solutions exist are to be presented in the next section. Figures 2 and 3 provide the surface plots (and better view) of $|u|$ for a vortex-free solution at $\Omega=0$ and a solution at $\Omega=20$ with four vortices, respectively. Each solution has a top and bottom view; the paraboloid shapes are easy to visualize from the pictures. In Fig. 4, we have plotted a solution with 19 vortices corresponding to $\Omega=31.5$. There is a triangular array with one vortex at the origin, then six vortices in the inner circle, and 12 vortices on the side.

\section{The case of an ellipse}

We now present some solutions for an ellipse corresponding to $\lambda=1.5$. In Fig. 5, the contour plots of the magnitude of the solution $|u|$ with $\Omega=17.5$ are drawn while in Fig. $6 \Omega$ $=25$. The rectangular domain showing in the individual pictures is of size 2.6 in the horizontal direction and 1.6 in the vertical direction. For $\Omega=17.5$, it is interesting to compare the location of vortices with the analysis of Sec. III C: in this case $\rho=0.79$, the long axis is 0.9887 , and we find for the location of the vortex $x=0.19$ for $n=2$ and $x=0.33$ for $n$ $=3$. The picture shows that the vortex is about $1 / 5$ of the long axis for two vortices and $1 / 3$ for three vortices, which is consistent with the analysis. We believe that, for moderate values of $n$, the location of $n$ vortices corresponds to the positions minimizing Eq. (3.5).
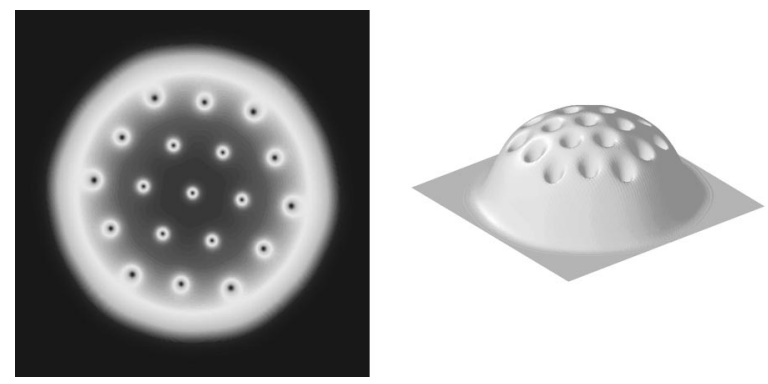

FIG. 4. Contour and surface plots of $|u|$ for solutions with 19 vortices at $\Omega=31.5$.
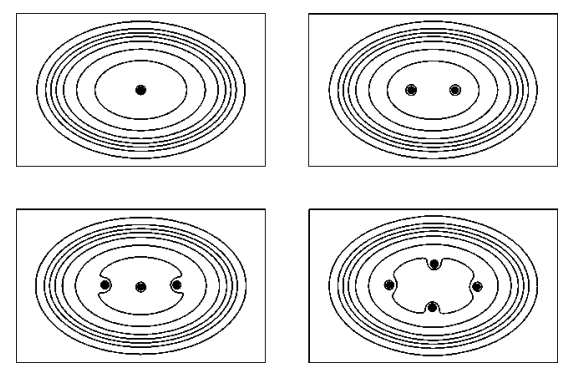

FIG. 5. Contour plots of $|u|$ for $\Omega=17.5, \lambda=1.5$.

Note that there were two vortex configurations with five vortices for $\Omega=25$, among which the nonsymmetric configuration corresponds to a solution with a lower free energy, although the difference in their energy values is very small. The coexistence of vortex solutions with the same number of vortices but with different vortex configurations has been experimentally observed in $[8,9]$.

\section{B. Branches of solutions}

An issue that we have studied is the existence of branches of $n$-vortex solutions as $\Omega$ is varied, and especially which one is the minimizer.

\section{The case of the disk}

For $\Omega=0$, the solution with lowest energy is the vortexfree solution. We start with this solution as initial value for the time-dependent problem for a slightly bigger $\Omega$. This device allows us to continue the branch of the vortex-free solution as $\Omega$ is increased. We find that the vortex-free solution is obtained as the limit of the evolution equation when $t$ is large up to $\Omega=19$. For $\Omega=19$, six vortices are nucleated from the boundary and eventually one vortex moves to the center and the final configuration is similar to that in Fig. 1 (top right). Now if $\Omega$ is decreased from the value 19 using the six-vortex solution as initial value, we see that this sixvortex solution branch exists down to $\Omega=16$, when it jumps to four vortices. If we decrease $\Omega$ further, then we stay on the four-vortex branch down to $\Omega=13$, when it drops to a two-vortex branch. Similarly, if we increase $\Omega$ from 13, the two-vortex solution will persist up to $\Omega=21$. As for the onevortex solution branch, it is computed by planting a vortexlike function at the center in the initial condition for $\Omega=10$; then the branch is computed by continuation in $\Omega$ (both increasing and decreasing). It is interesting to observe the fact that this branch extends all the way to $\Omega=0$; such persis-
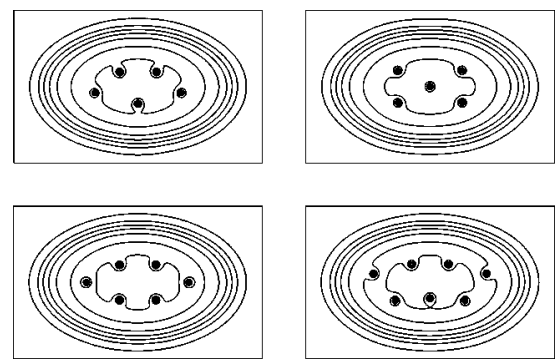

FIG. 6. Contour plots of $|u|$ for $\Omega=25, \lambda=1.5$. 
tence of the one-vortex solution even for zero velocity has been elaborated by various authors (see [4] for instance). Since implicit integration is used, we are indeed able to march to the steady state and to ascertain that this persistence is not due to metastability. On the other hand, if the vortex is planted away from the origin, it disappears for small $\Omega$ as we will see later.

\section{The case of an ellipse}

The same kind of behavior occurs for the vortex-free solution branch with $\lambda=1.5$ : when $\Omega$ is increased from 0 , we stay on the vortex-free branch up to $\Omega=22.5$ when the solution jumps to the four-vortex branch of Fig. 6. If we decrease $\Omega$ starting from this four-vortex branch, the solution will stay on it down to $\Omega=15$, when it jumps to two vortices and at $\Omega=10$ it jumps to the vortex-free solution. Similarly to the case of the disk, if one vortex is planted at the center at time 0 , it will persist in time even down to $\Omega=0$.

For $\Omega$ large enough, several vortices are nucleated at the same time. Both initial conditions and the round-off errors contribute to the symmetry breaking, and a strong symmetry presence often makes symmetry breaking much harder to achieve. For the disk case, we expect an equal chance of vortex nucleation from any point of the boundary. It turns out that, for $\Omega=19$, an unstable front produces oscillations of almost equal magnitude, and spins off six vortices at the same time. Had the disturbance being unevenly distributed, it would be possible for some vortices to get spun off ahead of others, and due to the repulsion the others may never have a chance to appear; thus we may see solutions with a smaller number of vortices. The value $\Omega=19$ corresponds to $\widetilde{\Omega} / \omega_{x}$ $=0.38$, which is less than 0.7 , for which vortices are nucleated in the experiment [9]. This may be due to our 2D simplification. For a theoretical investigation of this value, see [28]. Nevertheless, we believe that the location of vortices is well described by our theory.

\section{Energy diagrams}

We now discuss the energy diagrams in relation to the discussion of the critical angular velocities given in the earlier sections. In Figs. 7 and 8, we have plotted the energy given by Eq. (1.3) as a function of $\Omega$ for the various branches of solutions (according to the number of vortices). Not all computed solution branches are plotted since some of them are very close together. Again, $\varepsilon=0.02$ in our computation.

\section{The case of the disk}

As discussed earlier, the vortexless solution, in the case of the disk, exists for all values of $\Omega$ and is independent of $\Omega$; we thus have a constant line for its energy (see Fig. 7). These vortex-free solutions are the global energy minimizers for small $\Omega(\Omega<9.3)$ whereas for $\Omega>9.3$ they have larger energy than the one-vortex solution. For multivortex solutions, we see that each becomes the global energy minimizer for a range of values of $\Omega$, which corresponds to the experimental observation that there exist multivortex configurations for

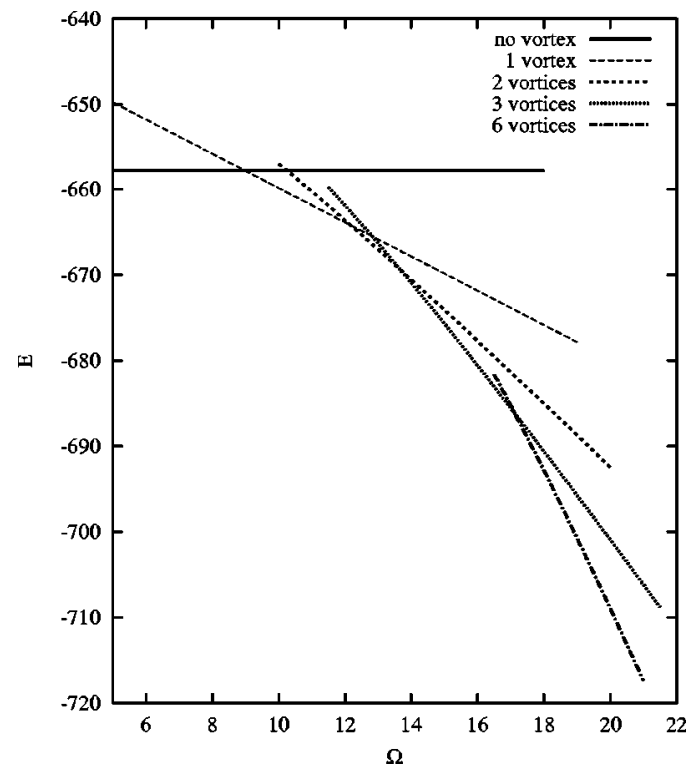

FIG. 7. The energy vs $\Omega$ curves for $\lambda=1.0$.

wide bands of angular velocities. It is interesting to compare this result with the value found in Sec. II where $\Omega_{1}=9.8$. Similarly, we obtain from Eq. (3.4) that $\Omega_{n}-\Omega_{n-1} \simeq 2.0$. Although the value of $\Omega_{1}$ is slightly overestimated, the difference $\Omega_{n}-\Omega_{n-1}$ looks good for small $n$ : the numerics indicate $\Omega_{2}=12.0, \Omega_{3}=13.6, \Omega_{4}=15.8$ and our theoretical computations yield $\Omega_{2}=11.8, \Omega_{3}=13.8, \Omega_{3}=15.8$.

However, when $\Omega$ is increased from 0 , we saw that we stay on the vortex-free branch up to $\Omega=19$. This means that the vortex-free solution is a local minimizer up to this value. We do not have any theoretical estimate for this value of local minimum. Similarly, for multivortex solutions, hysteresis loops are present. For the solution with six vortices, there are two possible configurations, one with all six on a concentric circle, and one with only five on a concentric

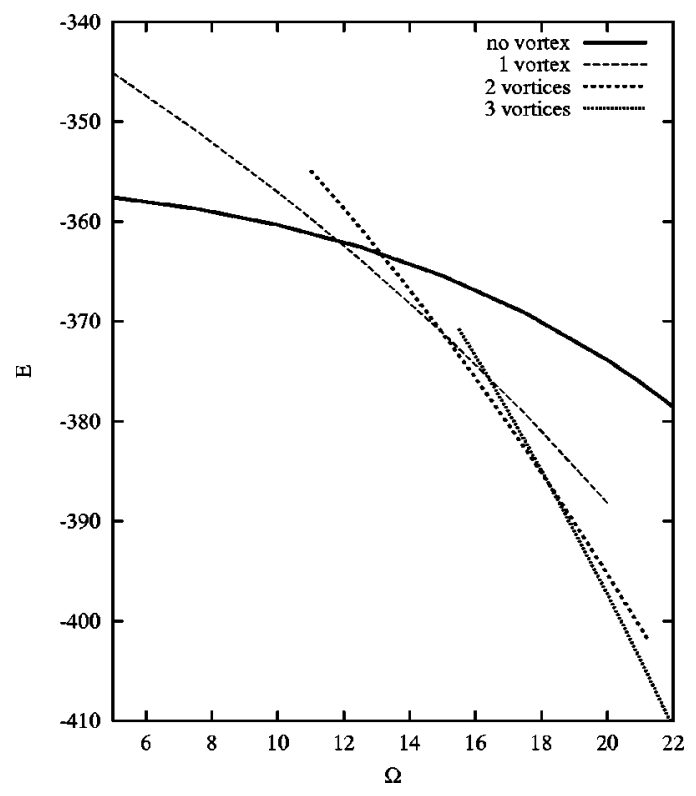

FIG. 8 . The energy vs $\Omega$ curves for $\lambda=1.5$. 

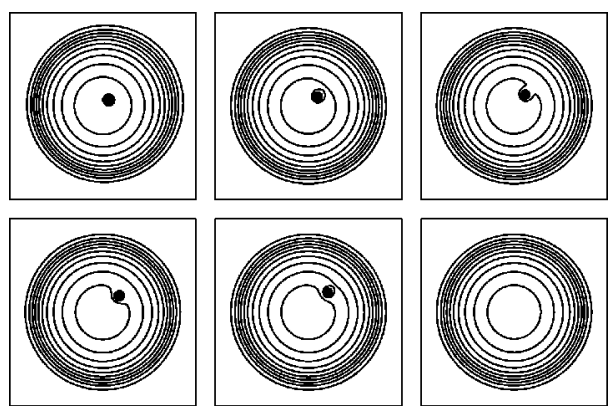

FIG. 9. Perturbing vortex away from center for $\Omega=0$.

circle while the remaining vortex is at the center of the disk. This occurs for $\Omega=22.5$. Although the difference in energy is hardly noticeable, the solution with a center vortex does have a smaller energy value.

\section{The case of an ellipse}

Now, we discuss the elliptical case with $\lambda=1.5$. The energy versus $\Omega$ curves are given in Fig. 8 .

The vortexless solution, in the case of the ellipse, is no longer independent of $\Omega$ as in the case of the disk, as illustrated by the dependence of its energy on $\Omega$. The energy diagram also illustrates that hysteresis occurs as a universal phenomenon both in the case of a disk and in the case of an ellipse.

Our numerics indicate that $\Omega_{1}=12, \Omega_{2}=15$. It is interesting to compare this result with the value found in Sec. II: we obtain from Eq. (3.4) that $\Omega_{1} \simeq 13$ and that $\Omega_{n}-\Omega_{n-1}$ $\simeq 2.88$. Although the value of $\Omega_{1}$ is slightly overestimated, the difference $\Omega_{n}-\Omega_{n-1}$ looks good again since we find $\Omega_{2}=14.9$.

\section{Displacement of the vortex from the center}

Based on the earlier estimate (3.1) for the one-vortex solution, we see that for small $\Omega$ the displacement of the vortex away from the center leads to the drop of energy $(\Omega<4.9)$. For slightly bigger $\Omega$ a vortex at the center is a local minimum $(\Omega<9.8)$. Let us analyze the time-dependent equation using a vortex off center as the initial condition and let us examine how it evolves. We find that in the case of the disk, for $\Omega<7$, a displacement of size one-tenth of the radius causes the displaced center vortex in the one-vortex solution to move toward the boundary. For $\Omega>10$, the center vortex in the one-vortex solution moves back toward the center if under a displacement of size one-third of the radius. For
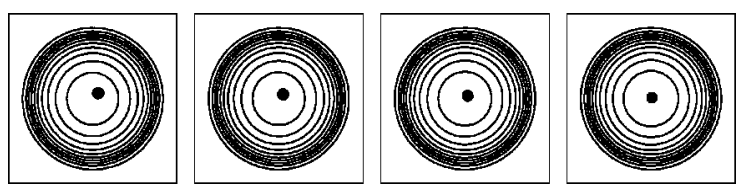

FIG. 10. Perturbing vortex away from center for $\Omega=7.9$.

intermediate values of $\Omega$, the vortex moves back to the center for small displacement but moves toward the boundary for large displacement. Figures 9 and 10 show, in the case of a disk and a small displacement, the marching away of the center vortex for the solution with $\Omega=0$ (starting from the top row and then to the bottom row) and the marching back to the center for the solution with $\Omega=7.9$.

\section{CONCLUSION}

We have presented an alternative framework for the study of the Gross-Pitaevskii energy in the Thomas-Fermi limit: we defined a nondimensionalized parameter $\varepsilon$ and computed theoretically an asymptotic expansion of the energy, the critical thermodynamic angular velocities of nucleation of vortices, and the location of vortices. This extends the results of [2]. We have proposed and implemented time integration schemes that have norm-preserving and energy-decreasing features and thus are useful for studying the stability and metastability of solutions. We also presented energy diagrams computed numerically for the various vortex solutions. We determined that our theoretical predictions for critical thermodynamic angular velocities are quite consistent with the numerics, which encourages us to think that our approximation of small $\varepsilon$ is correct although $|\ln \varepsilon|$ is not small. In our computation, we took $\varepsilon=0.02$, and the number of vortices we observed ranges from 0 to 19 for $\Omega$ between 0 and 31.5.

In very recent work [29], we used this framework of study and the results in [30] for the Ginzburg-Landau free energy in $3 \mathrm{D}$ to get results for rotating Bose-Einstein condensates in 3D, especially estimates of critical angular velocities and an asymptotic expansion of the energy that is valid for any type of vortex line. We will also carry out further numerical simulations in $3 \mathrm{D}$ to compare with experimental data.

\section{ACKNOWLEDGMENTS}

The authors are grateful to Y. Castin for very interesting discussions. They would also like to thank T. Riviere, E. Sandier, and S. Serfaty. This work is supported in part by a joint France-Hong Kong research grant.
[1] D. Butts and D. Rokhsar, Nature (London) 397, 327 (1999).

[2] Y. Castin and R. Dum, Eur. Phys. J. D 7, 399 (1999).

[3] F. Dalfovo, S. Giorgini, L. Pitaevskii, and S. Stringari, Rev. Mod. Phys. 71, 463 (1999).

[4] D.L. Feder, C.W. Clark, and B.I. Schneider, Phys. Rev. Lett. 82, 4956 (1999).

[5] A.L. Fetter, Phys. Rev. 140, A429 (1965).
[6] A.L. Fetter and A.A. Svidzinsky, e-print cond-mat/0102003.

[7] M.R. Matthews et al., Phys. Rev. Lett. 83, 2498 (1999).

[8] K.W. Madison, F. Chevy, W. Wohlleben, and J. Dalibard, Phys. Rev. Lett. 84, 806 (2000).

[9] K.W. Madison, F. Chevy, W. Wohlleben, and J. Dalibard, J. Mod. Opt. 47, 2715 (2000). 
[10] A.A. Svidzinsky and A.L. Fetter, Phys. Rev. Lett. 84, 5919 (2000).

[11] R. Onofrio et al., Phys. Rev. Lett. 85, 2228 (2000).

[12] J.S. Stiessberger and W. Zwerger, Phys. Rev. A 62, 061601 (2000).

[13] A. Aftalion, E. Sandier, and S. Serfaty, J. Math. Pures Appl. 80, 339 (2001).

[14] S. Serfaty, Commun. Contemp. Math. 1, 213 (1999); 1, 295 (1999).

[15] S. Serfaty, ESAIM Control Optim. Calc. Var. 6, 201 (2001). (1999).

[16] F. Dalfovo, L. Pitaevskii, and S. Stringari, Phys. Rev. A 54, 4213 (1996).

[17] A.L. Fetter and D.L. Feder, Phys. Rev. A 58, 3185 (1998).

[18] F. Bethuel, H. Brezis, and F. Helein, Ginzburg-Landau Vortices (Birkhäuser, Boston, 1994).

[19] F. Bethuel and T. Riviere, Ann. Inst. Henri Poincare, Anal. Non Lineaire 12, 243 (1995).

[20] E. Lundh, e-print cond-mat/0103272.
[21] D.L. Feder, C.W. Clark, and B.I. Schneider, Phys. Rev. A 61, 011601(R) (1999).

[22] S. Chapman, Q. Du, M. Gunzburger, and J. Peterson, Adv. Math. Sci. Appl. 5, 193 (1995).

[23] Q. Du, M.D. Gunzburger, and J.S. Peterson, Phys. Rev. B 46, 9027 (1992); 51, 16194 (1995).

[24] F. Lin and Q. Du, SIAM (Soc. Ind. Appl. Math.) J. Math. Anal. 28, 1265 (1997).

[25] L. Simon, Ann. Math. 118, 525 (1983).

[26] Q. Du and P. Gray, SIAM (Soc. Ind. Appl. Math.) J. Appl. Math. 56, 1060 (1996).

[27] Q. Du, M.D. Gunzburger, and J.S. Peterson, SIAM Rev. 34, 54 (1992).

[28] S. Sinha and Y. Castin, e-print cond-mat/0101292.

[29] A. Aftalion and T. Riviere, e-print cond-mat/0105208.

[30] F. Pacard and T. Riviere, in Progress in Nonlinear Differential Equations and their Applications (Birkhäuser, Boston, 2000), Vol. 39. 